\title{
Tratamento de sementes sobre a germinação, o vigor e o desenvolvimento do trigo
}

\author{
Seed treatment on germination, vigor and development of wheat
}

\author{
Fernando Sartori Pereira ${ }^{1}$, Lucas Antonio Stempkowski ${ }^{1}$, Juliana Borba Valente ${ }^{1}$, Paulo Roberto \\ Kuhnem $^{2}$, Douglas Lau ${ }^{3}$, Ricardo Trezzi Casa ${ }^{1}$ e Fabio Nascimento da Silva ${ }^{1 *}$ \\ 1'Universidade do Estado de Santa Catarina, Lages, SC, Brasil. *Autor para correspondência: fabio.silva@udesc.br \\ ${ }^{2}$ Biotrigo Genética, Passo Fundo, RS, Brasil. \\ ${ }^{3}$ Embrapa Trigo, Passo Fundo, RS, Brasil.
}

Submissão: 10/10/2018 / Aceite: 05/04/2019

\begin{abstract}
RESUMO
O tratamento de sementes (TS) é uma importante ferramenta no manejo de doenças na cultura do trigo, embora poucos produtos sejam indicados para esse fim. Por isso, a pesquisa deve realizar trabalhos relacionados à efetividade de diferentes produtos no TS e também o possível efeito fitotóxico. Os objetivos deste trabalho foram realizar testes fisiológicos em sementes e avaliar o desenvolvimento de duas cultivares de trigo (BRS Guamirim e TBIO Toruk), submetidas a 13 diferentes tratamentos de sementes. Para cada tratamento foram analisados a germinação e o vigor. As sementes tratadas também foram semeadas a campo, onde se determinou a emergência a campo e massa fresca de raiz e parte aérea. As sementes tratadas com azoxistrobina e Bacillus amyloliquefaciens resultaram em decréscimo na germinação da cultivar TBIO Toruk. Na análise do vigor houve diferença entre os tratamentos testados para as duas cultivares, sendo que houve redução do vigor em sementes tratadas com dimetomorfe para TBIO Toruk e azoxistrobina para a cultivar BRS Guamirim. As sementes submetidas ao tratamento Carboxamida A resultaram em plântulas com maiores valores de massa fresca de raiz e parte aérea. Os resultados apresentados permitem a seleção de produtos com baixa ou ausência de fitotoxidade para incorporação no manejo de doenças no trigo.
\end{abstract}

PALAVRAS-CHAVE: Triticum aestivum, fungicidas, agentes de biocontrole, indutor de resistência, fitotoxicidade.

\begin{abstract}
Seed treatment (ST) is an important tool in the management of diseases in the wheat crop, although few products are indicated for this purpose. The research must carry out studies related to the ST effectiveness using different products and the possible phytotoxic effect. The aim of this study was to perform physiological tests on seeds and evaluate the development of two wheat cultivars (BRS Guamirim and TBIO Toruk), submitted to 13 different ST. Germination and vigor were analyzed for each treatment. The treated seeds were also sown to the field, where the field emergence and fresh root and shoot mass were determined. In the germination test, the seeds treated azoxystrobin and Bacillus amyloliquefaciens presented a decrease in the cultivar TBIO Toruk. Vigor analyzes indicated differences between treatments in the two cultivars, and the dimethomorph treatment presented the lowest vigor for TBIO Toruk and azoxystrobin for the BRS Guamirim. The seeds submitted to the Carboxamide A treatment showed seedlings the highest values of fresh root and shoot mass. The results presented allow the selection of products with low or no phytotoxicity for incorporating in wheat disease management.
\end{abstract}

KEYWORDS: Triticum aestivum, fungicides, biocontrol agents, resistance inducer, phytotoxicity.

A utilização de tratamento de sementes é uma prática importante para redução de patógenos que infestam e infectam as sementes, além da proteção contra patógenos que sobrevivem no solo quando é realizada a semeadura (HENNING 2005). No tratamento de sementes diversos produtos podem ser utilizados, tais como, agroquímicos (fungicidas e inseticidas), produtos biológicos, inoculantes, estimulantes e micronutrientes (MENTEN \& MORAES 2010). O tratamento químico baseia-se na existência de produtos eficientes contra os patógenos, que apresentem baixa fitotoxicidade e sejam pouco tóxicos ao homem e ao ambiente (MENTEN 1991). O tratamento de sementes de trigo é essencial, tendo em vista a redução de 
inóculo de patógenos que são vinculados às sementes, favorecendo assim aspectos fisiológicos das sementes e de desenvolvimento das plântulas. A utilização de sementes de elevada qualidade fisiológica e sanitária pode favorecer o rendimento de grãos nas lavouras de trigo (LIMA et al. 2006).

De acordo com MARCOS FILHO (2017), vigor é o conjunto de atributos que confere à semente a capacidade para germinar, emergir e resultar rapidamente em plântulas normais, mesmo com ampla diversidade das condições do ambiente. A germinação das sementes pode sofrer alterações por fatores internos, relacionados à longevidade, vigor e também por fatores externos, como água, temperatura e oxigênio (CARVALHO \& NAKAGAWA 2012). Resultados de pesquisas, embora escassos, têm evidenciado que alguns produtos, quando aplicados às sementes, podem, em determinadas situações, ocasionar redução na germinação destas e na sobrevivência das plântulas (NASCIMENTO et al. 1996).

O objetivo desse estudo foi avaliar os efeitos de diferentes tratamentos sobre a qualidade fisiológica de sementes de trigo e sobre o desenvolvimento das plantas no campo. Para isso, sementes das cultivares TBIO Toruk e BRS Guamirim, foram tratadas em sacos plásticos, adicionando-se os produtos e agitadas manualmente até a cobertura das mesmas. No tratamento testemunha foi utilizado apenas água destilada + polímero. As formulações, concentrações e doses em $\mathrm{g}$ ou $\mathrm{mL}$ de p.c./100kg de sementes utilizadas estão descritos a seguir: (I) testemunha; (ii) Bacillus subtilis 13,68 g/L (10); (iii) Trichoderma asperellum (mínimo 1 x $10^{10} \mathrm{UFC} / \mathrm{g}$ ) 28\% (100); (iv) Acibenzolar-S- Metílico $500 \mathrm{~g} / \mathrm{kg}$ (25); (v) Azoxistrobina $250 \mathrm{~g} / \mathrm{L}$ (200); (vi) Piraclostrobina 25g/L + Tiofanato Metílico 225g/L+ Fipronil $250 \mathrm{~g} / \mathrm{L}$ (200); (vii) Tiofanato Metílico $350 \mathrm{~g} / \mathrm{L}$ + Fluazinam 52,5 g/L (200); (viii) Fenamidona $500 \mathrm{~g} / \mathrm{L}$ (200); (ix) Fludioxonil 37,5 g/L + Metalaxil- M $30 \mathrm{~g} / \mathrm{L}$ + Tiabendazole $300 \mathrm{~g} / \mathrm{L}$ + Azoxistrobina $15 \mathrm{~g} / \mathrm{L} \mathrm{(100);} \mathrm{(x)} \mathrm{Carboxamida} \mathrm{A} 330 \mathrm{~g} / \mathrm{L} \mathrm{(45);} \mathrm{(xi)} \mathrm{Bacillus}$ amyloliquefaciens (2 x1010 UFC/g) (10); (xi) Metalaxil $350 \mathrm{~g} / \mathrm{L}(5,72)$; e (xiii) Dimetomorfe $500 \mathrm{~g} / \mathrm{Kg}(80)$.

O teste de germinação foi realizado conforme descrito nas Regras de Análise de Sementes (RAS) (BRASIL 2009). Para o teste de vigor, seguiu-se a metodologia descrita por MARCOS FILHO (1999), utilizando o teste de envelhecimento acelerado. Ambos os testes fisiológicos, foram realizados com quatro repetições de 100 sementes por tratamento. As plântulas normais foram avaliadas de acordo com a RAS (BRASIL 2009), em delineamento experimental inteiramente casualizado.

Para a determinação de plantas emersas foi utilizado o delineamento em blocos casualizados, com cinco repetições. A semeadura ocorreu em 04 de julho de 2017 em Passo Fundo, RS, sendo realizada a abertura de sulcos, adubação com $300 \mathrm{~kg} / \mathrm{ha}$ da fórmula 5-25-25 (N- $\left.\mathrm{P}_{2} \mathrm{O}_{5}-\mathrm{K}_{2} \mathrm{O}\right)$ e semeadura manual. Cada tratamento foi composto por duas linhas de $0,5 \mathrm{~m}$ de comprimento com 36 sementes/linha. O número de plantas normais emergidas foi determinado 16 dias após a semeadura (DAS) por contagem manual em cada parcela do ensaio, sendo realizada 10 dias após a emergência (DAE) das plântulas.

Aos 42 dias DAE foi realizado uma avaliação da massa fresca de raiz e parte aérea nas linhas destinadas as coletas de amostras. Foram coletadas duas plantas com o sistema radicular por tratamento e cultivar de cada repetição, sendo essas escolhidas ao acaso nos cinco blocos. Posteriormente, as raízes foram lavadas em água corrente, divididas em parte aérea e raiz e pesadas em balança de precisão, sendo o resultado expresso em gramas.

Os dados obtidos foram analisados estatisticamente pela análise de variância, utilizando o pacote estatístico ExpDes.pt (FERREIRA et al. 2013) implementado no software R versão 3.4.1 (R Foundation for Statistical Computing, Vienna, Austria).

No teste de germinação em rolo de papel (Tabela 1), os tratamentos apresentaram desempenho satifatório, conforme os padrões para produção e comercialização, que estabelecem valores mínimos de $80 \%$ de germinação para semente certificada de primeira geração (C1) e certificada de segunda geração (C2), semente de primeira geração (S1) e semente de segunda geração (S2) (MAPA 2013). Para germinação da cultivar TBIO Toruk o tratamento testemunha apresentou (95\%), diferindo dos tratamentos azoxistrobina (87\%) e B. amyloliquefaciens (88\%). Não houve diferença significativa para a germinação entre os tratamentos testados na cultivar BRS Guamirim, com a germinação variando de 90 a $96 \%$. Segundo COSTA et al. (2018) alguns produtos quando aplicados nas sementes podem, em determinadas situações, ocasionar redução na germinação e na sobrevivência das plântulas. Ao analisar a cultivar de trigo Pampeano e Quartzo, HOSSEN et al. (2014), verificaram que não houve diferença significativa entre o tratamento controle, carboxina + thiram, tiametoxam + carboxina + thiram, para o percentual de germinação.

Para o teste de envelhecimento acelerado (Tabela 1), constatou-se redução do vigor, quando comparada ao teste de germinação convencional. $O$ teste apresentou diferença entre os tratamentos testados. Para a cultivar TBIO Toruk a porcentagem de vigor das sementes tratadas com: tiofanato metílico + fluazinam, piraclostrobina + tiofanato metílico + fipronil, Carboxamida A e tratamento controle, apresentaram porcentagens superiores $(88 ; 89 ; 79$ e $84 \%$, respectivamente) não difererindo 
estatisticamente entre si; enquanto as sementes tratadas com: B. amyloliquefaciens, metalaxil e dimetomorfe reduziram o vigor (62; 56 e 50\%, respectivamente). Para BRS Guamirim as sementes dos tratamentos testemunha, B. subtilis, acibenzolar-s-metílico, piraclostrobina + tiofanato metílico + fipronil, tiofanato metílico + fluazinam, fludioxonil + metalaxil-m + tiabendazole + azoxistrobina e carboxamida A não diferiram, apresentando alto vigor (valores entre 86 a 95\%). No trabalho de HOSSEN et al. (2014) foi observado para o teste de envelhecimento acelerado, que o melhor tratamento foi o tiametoxam + carboxina + thiram, quando comparado com a testemunha sem aplicação, não diferindo do tratamento tiametoxam + thiram, para as cultivares Quartzo e Pampeano. Neste trabalho, as sementes tratadas com o tratamento piraclostrobina + tiofanato metílico + fipronil apresentou alta porcentagem de germinação e vigor. Sendo observado $92 \%$ de germinação, $89 \%$ de vigor na cultivar Toruk e $95 \%$ de germinação e $91 \%$ de vigor para a cultivar BRS Guamirim.

Tabela 1. Porcentagem de germinação e vigor de sementes de trigo das cultivares TBIO Toruk e BRS Guamirim, em função da aplicação de fungicida, indutor de resistência e agentes de biocontrole em tratamentos de sementes.

Table 1. Germination percentage and vigor of the seed wheat cultivars TBIO Toruk and BRS Guamirim, according to the fungicides, biocontrol agents and resistance inducer application in seed treatments.

\begin{tabular}{lcccc}
\hline \multirow{2}{*}{\multicolumn{1}{c}{ Tratamentos }} & \multicolumn{2}{c}{ Germinação (\%) } & \multicolumn{2}{c}{ Vigor (\%) } \\
\cline { 2 - 5 } & TBIO Toruk & $\begin{array}{c}\text { BRS } \\
\text { Guamirim }\end{array}$ & TBIO Toruk & $\begin{array}{c}\text { BRS } \\
\text { Guamirim }\end{array}$ \\
\hline Testemunha sem aplicação & $95 \mathrm{a}$ & $92 \mathrm{~ns}$ & $84 \mathrm{ab}$ & $88 \mathrm{abc}$ \\
Bacillus subtilis & $91 \mathrm{ab}$ & 92 & $64 \mathrm{def}$ & $91 \mathrm{abc}$ \\
Trichoderma asperellum & $91 \mathrm{ab}$ & 94 & $73 \mathrm{bcd}$ & $84 \mathrm{bc}$ \\
Acibenzolar-S- Metílico & $89 \mathrm{ab}$ & 90 & $64 \mathrm{def}$ & $86 \mathrm{abc}$ \\
Azoxistrobina & $87 \mathrm{~b}$ & 91 & $73 \mathrm{dcd}$ & $74 \mathrm{~d}$ \\
Piraclostrobina + Tiofanato Metílico + Fipronil & $92 \mathrm{ab}$ & 95 & $89 \mathrm{a}$ & $91 \mathrm{abc}$ \\
Tiofanato Metílico + Fluazinam & $91 \mathrm{ab}$ & 93 & $88 \mathrm{a}$ & $95 \mathrm{a}$ \\
Fenamidona & $88 \mathrm{ab}$ & 94 & $76 \mathrm{bc}$ & $84 \mathrm{bc}$ \\
Fludioxonil + Metalaxil-M + Tiabendazole + & $90 \mathrm{ab}$ & 93 & $69 \mathrm{cde}$ & $91 \mathrm{abc}$ \\
Azoxistrobina & $89 \mathrm{ab}$ & 96 & $79 \mathrm{abc}$ & $93 \mathrm{ab}$ \\
Carboxamida A & $88 \mathrm{~b}$ & 90 & $62 \mathrm{ef}$ & $86 \mathrm{bc}$ \\
Bacillus amyloliquefaciens & $91 \mathrm{ab}$ & 94 & $56 \mathrm{fg}$ & $84 \mathrm{bcd}$ \\
Metalaxil & $88 \mathrm{ab}$ & 92 & $50 \mathrm{~g}$ & $83 \mathrm{~cd}$ \\
Dimetomorfe & 3,0 & 2,7 & 6,0 & 4,5 \\
\hline CV (\%) & &
\end{tabular}

Médias seguidas pelas mesmas letras na coluna não diferem significativamente pelo teste de Tukey $(p<0,05)$.

Para plantas emersas, na cultivar TBIO Toruk os tratamentos não apresentaram diferença significativa $(p<0,05)$ (Tabela 2). Para a cultivar BRS Guamirim, as sementes tratadas com $T$. asperellum e azoxistrobina apresentaram as maiores porcentagens de plantas emersas, diferindo estatisticamente do tratamento fludioxonil + metalaxil + tiabendazole + azoxistrobina, possivelmente devido a fitotoxicidade dos fungicidas. Em trabalho realizado em casa de vegetação utilizando a cultivar de trigo Fundacep Horizonte, não foram encontradas diferenças na emergência de plantas entre os tratamentos de sementes utilizando os fungicidas triadimenol $\left(25,11 \mathrm{~g}\right.$ i.a. ha- ${ }^{-1}$, carboxina + tiram ( $75 \mathrm{~g}$ i.a. ha-1) e testemunha (ULGUIM et al. 2013).

Na massa fresca da parte aérea da cultivar TBIO Toruk as plântulas dos tratamentos testemunha, $T$. asperellum, azoxistrobina, piraclostrobina + tiofanato metílico + fipronil, fludioxonil + metalaxil + tiabendazole + azoxistrobina e Carboxamida A apresentaram maiores valores de massa fresca da parte aérea, porém não apresentaram diferença significativa. Os menores valores de massa fresca da parte aérea foram observados em plântulas dos tratamentos fenamidona $(3,2 \mathrm{~g})$, que diferenciou da testemunha. Esse comportamento possivelmente foi devido ao efeito de fitotoxicidade para as plântulas de trigo nos estádios iniciais. Para massa fresca de raiz na cultivar TBIO Toruk, plântulas dos tratamentos acibenzolar-s-metílico, piraclostrobina + tiofanato metílico + fipronil, tiofanato metílico + fluazinam e Carboxamida A apresentaram 
as maiores massas, enquanto que os tratamentos azoxistrobina, fenamidona e metalaxil as menores massas de raiz (Tabela 2). Para a cultivar BRS Guamirim os melhores resultados de massa fresca da parte aérea foram em plântulas dos tratamentos testemunha, acibenzolar-s-metílico, tiofanato + fluazinam, Carboxamida $A$ e dimetomorfe, sendo que os menores valores foram observados nos tratamentos azoxistrobina, $B$. subtilis, testemunha, piraclostrobina + tiofanato metílico + fipronil, fenamidona e metalaxil. Para massa fresca da raiz, a cultivar BRS Guamirim apresentou as maiores massas nos tratamentos testemunha, azoxistrobina e dimetomorfe, enquando os tratamentos $B$. subtilis e $B$. amyloliquefaciens apresentaram as menores massas fresca de raiz (Tabela 1). No trabalho realizado em casa de vegetação, com as cultivares de trigo Mirante e CD 104, utilizando os fungicidas difenoconazole e carboxin + thiram, nas doses de $30+50+50 \mathrm{~g}$ por $100 \mathrm{~kg}$ de sementes do ingrediente ativo, respectivamente, ISHIKAWA et al. (2012) observaram aos 41 dias após a semeadura que a testemunha foi superior em acúmulo de massa fresca de raiz e parte aérea, diferindo dos tratamentos difenoconazole e carboxin + thiram. Segundo os autores, esse comportamento pode ser explicado por um possível efeito fitotóxico dos produtos no desenvolvimento das plantas. Esses dados corroboram com o que foi descrito nesse trabalho, sendo que a testemunha, em alguns casos, não diferiu da maioria dos tratamentos.

Tabela 2. Plantas emersas a campo e massa fresca de raiz e parte aérea 42 dias após emergência das cultivares de trigo TBIO Toruk e BRS Guamirim, em função da aplicação de fungicidas, agentes de biocontrole e indutor de resistência em tratamentos de sementes.

Table 2. Plants emersed and fresh root and shoot mass 42 days after emergence of the wheat cultivars TBIO Toruk and BRS Guamirim, according to the fungicides, biocontrol agents and resistance inducer application in seed treatments.

\begin{tabular}{|c|c|c|c|c|c|c|}
\hline \multirow[b]{2}{*}{ Tratamentos } & \multicolumn{4}{|c|}{ TBIO Toruk } & \multicolumn{2}{|c|}{ BRS Guamirim } \\
\hline & PE & $\begin{array}{c}\text { MFPA } \\
\text { (gramas) }\end{array}$ & $\begin{array}{c}\text { MFR } \\
\text { (gramas) }\end{array}$ & PE & $\begin{array}{c}\text { MFPA } \\
\text { (gramas) }\end{array}$ & $\begin{array}{c}\text { MFR } \\
\text { (gramas) }\end{array}$ \\
\hline Testemunha sem aplicação & 29,4 ns & $5,5 a b$ & 0,7 cde & $31,6 \mathrm{ab}$ & $6,0 \mathrm{~cd}$ & $0,9 a b c$ \\
\hline Bacillus subtilis & 28,6 & $4,3 \mathrm{bcd}$ & $0,8 \mathrm{bcd}$ & $29,4 a b$ & $4,9 \mathrm{~cd}$ & $0,6 \mathrm{e}$ \\
\hline Trichoderma asperellum & 29,2 & $5,2 a b c$ & 0,7 cde & $33,2 \mathrm{a}$ & $5,0 \mathrm{~cd}$ & $0,8 \mathrm{abcd}$ \\
\hline Acibenzolar-S- Metílico & 28,6 & $4,4 \mathrm{bcd}$ & $0,8 \mathrm{abc}$ & $32,0 \mathrm{ab}$ & $5,8 \mathrm{abcd}$ & 0,7 cde \\
\hline Azoxistrobina & 29,2 & $5,0 a b c$ & $0,7 \mathrm{de}$ & $33,2 \mathrm{a}$ & $4,9 \mathrm{~cd}$ & $1,0 \mathrm{a}$ \\
\hline $\begin{array}{l}\text { Piraclostrobina + Tiofanato } \\
\text { Metílico + Fipronil }\end{array}$ & 29,8 & $5,6 \mathrm{ab}$ & $0,9 a b$ & $32 \mathrm{ab}$ & $4,6 \mathrm{~cd}$ & $0,9 \mathrm{abcd}$ \\
\hline Tiofanato Metílico + Fluazinam & 28,8 & $4,4 \mathrm{bcd}$ & $1,0 \mathrm{a}$ & $32,6 a b$ & $5,9 \mathrm{abcd}$ & $0,9 \mathrm{abcd}$ \\
\hline Fenamidona & 27,2 & $3,2 d$ & $0,6 \mathrm{e}$ & $31,6 a b$ & $4,7 \mathrm{~cd}$ & $0,8 \mathrm{abcd}$ \\
\hline $\begin{array}{l}\text { Fludioxonil }+ \text { Metalaxil- } M+ \\
\text { Tiabendazole }+ \text { Azoxistrobina }\end{array}$ & 30,2 & $5,0 \mathrm{abc}$ & 0,8 bcde & $27,8 b$ & $5,5 \mathrm{bcd}$ & 0,8 bcde \\
\hline Carboxamida A & 29,0 & $5,9 \mathrm{a}$ & $0,9 a b$ & $31,0 a b$ & $6,9 a b$ & $0,8 \mathrm{abcd}$ \\
\hline Bacillus amyloliquefaciens & 32,6 & $3,9 \mathrm{~cd}$ & 0,7 cde & $32,2 \mathrm{ab}$ & $5,1 \mathrm{~cd}$ & $0,7 \mathrm{de}$ \\
\hline Metalaxil & 31,2 & $4,3 \mathrm{bcd}$ & $0,7 \mathrm{de}$ & $31,8 a b$ & $4,4 d$ & 0,8 bcde \\
\hline Dimetomorfe & 29,4 & $4,56 \mathrm{bc}$ & 0,7 cde & $31,2 a b$ & $7,2 \mathrm{a}$ & $1,0 \mathrm{ab}$ \\
\hline $\mathrm{CV}(\%)$ & 7,9 & 12,3 & 9,9 & 7,2 & 13,3 & 11,3 \\
\hline
\end{tabular}

PE - plantas emersas; MFPA - massa fresca da parte aérea; MFR - massa fresca de raíz.

Médias seguidas pelas mesmas letras na coluna não diferem significativamente pelo teste de Tukey $(p<0,05)$.

As doses utilizadas apresentaram resultados satisfatórios para germinação, as sementes tratadas com azoxistrobina e B. amyloliquefaciens reduziram a germinação para a cultivar TBIO Toruk em comparação a testemunha, mas ficaram acima dos $80 \%$, parâmetro de comercialização para semente. Maiores diferenças entre os tratamentos foram observadas no teste de vigor, onde 0 tratamento azoxistrobina reduziu a germinação das sementes. Analisando as duas cultivares, as sementes tratadas com piraclostrobina + tiofanato Metílico + fipronil, tiofanato metílico + fluazinam e Carboxamida A apresentaram os maiores percentuais de vigor, enquanto metalaxil e dimetomorfe nas doses utilizadas houve as maiores reduções de vigor. De um modo geral, as sementes tratadas com agentes de biocontrole não apresentaram dados satisfatórios de incremento para massa fresca de raiz e parte aérea. Os produtos avaliados que não afetaram a qualidade fisiológica das sementes, mostraram ter potencial para tratamento de sementes, e poderiam ser incorporados em programas de manejo de doenças do trigo. 


\section{AGRADECIMENTOS}

Os autores agradecem a Universidade do Estado de Santa Catarina (UDESC), Coordenação de Aperfeiçoamento de Pessoal de Nível Superior (CAPES), Biotrigo Genética e EMBRAPA Trigo, pelo amparo a pesquisa e auxílio financeiro.

\section{REFERÊNCIAS}

BRASIL. 2009. Ministério da Agricultura, Pecuária e Abastecimento. Regras para análise de sementes. Brasília: Secretaria de Defesa Agropecuária. 399p.

CARVALHO NM \& NAKAGAWA J. 2012. Sementes: Ciência, Tecnologia e Produção. 5.ed. Jaboticabal: FUNEP. 588p.

COSTA EM et al. 2018. Efeito fisiológico de inseticidas e fungicida sobre a germinação e vigor de sementes de soja (Glycine max I.). Científic@ Multidisciplinary Journal 5: 77-84.

FERREIRA EB et al. 2013. ExpDes.pt: Experimental Designs pacakge (Portuguese). R package version 1.1.2.

HENNING AA. 2005. Patologia e tratamento de sementes: noções gerais. 2.ed. Londrina: Embrapa. p.1-52.

HOSSEN DC et al. 2014. Tratamento químico de sementes de trigo. Pesquisa Agropecuária Tropical 44: 104-109.

ISHIKAWA MS et al. 2012. Tratamento químico de sementes sobre o desenvolvimento de mancha marrom em plantas de trigo. Ciência Rural 42: 1341-1346.

LIMA TC et al. 2006. Avaliação do vigor de trigo pelo teste de envelhecimento acelerado. Revista Brasileira de Sementes 28: 106-113.

MAPA. 2013. Ministério da Agricultura, Pecuária e Abastecimento. Instrução Normativa Mapa 45/2013. Brasília: MAPA. 22p.

MARCOS FILHO J. 2017. Conceituação do vigor de sementes em seus múltiplos aspectos. In: XX Congresso brasileiro de semente. Resumos...Londrina: ABRATES. p. 28.

MARCOS FILHO J. 1999. Teste de envelhecimento acelerado. In: KRZYZANOWSKI FC et al. (Eds.). Vigor de sementes: conceitos e testes. Londrina: Abrates. p.1-24.

MENTEN JOM. 1991. Importância do tratamento de sementes. In: MENTEN JOM. Patógenos em sementes: detecção, danos e controle químico. Piracicaba: ESALQ/FEALQ. p. 203-224.

MENTEN JO \& MORAES MHD. 2010. Tratamento de sementes: Histórico, tipos, características e benefício. Londrina: Abrates. p. 52-71.

NASCIMENTO WMO et al. 1996. Fitotoxidade do inseticida carbofuran 350 FMC na qualidade fisiológica de sementes de milho. Revista Brasileira de Sementes 18: 242-245.

ULGUIM AR et al. 2013. Características agronômicas do trigo em função do tratamento de sementes e épocas de aplicação de nitrogênio. Pesquisa Agropecuária Gaúcha 19: 16-23. 